\title{
Measurement of Anaerobic Threshold During Dynamic Exercise in Healthy Subjects: Comparison Among Visual Analysis and Mathematical Models
}

\author{
JC Crescêncio, LEB Martins*, LO Murta Jr, CM Antloga, RT Kozuki, MDB Santos, JA Marin Neto, \\ BC Maciel, L Gallo Jr
}

\author{
Division of Cardiology, Medical School of Ribeirão Preto, University of São Paulo, Ribeirão Preto \\ and *State University of Campinas, Campinas, Brazil
}

\begin{abstract}
The anaerobic threshold (AT) during dynamic physical exercise is a good parameter to quantify oxygen uptake and transport which reflects the functional cardiorespiratory reserve in men. The increasing use of computerized ergospirometric systems has allowed recording, processing and plotting ventilatory variables, cycle by cycle, on a real time basis. So, response changes in carbon dioxide production $\left(\mathrm{VCO}_{2}\right)$ may be used as a non-invasive measurement of AT. In the present study a custom software was developed to apply bi-segmental models (linear-linear and linear-quadratic) using the least square method for fitting $\mathrm{VCO}_{2}$ data. The lowest value of the residual sum of squares found in $\mathrm{VCO}_{2}$ graphic plots corresponded to AT, expressed as oxygen uptake. Data analysis showed that, despite the good correlation documented between the linear-linear model and the classical visual method, the mathematical method underestimates the AT values when compared to the visual one.
\end{abstract}

\section{Introduction}

During dynamic physical exercise executed at progressively increasing power intensities, the anaerobic threshold (AT) signals the point where an important change occurs in the physiological status of the organism as a whole, i.e., the beginning of anaerobic metabolism in muscles in contractile activity. This condition, associated with other processes already underway during exercise, such as aerobic metabolism, permits the progressive formation of chemical energy which is stored as adenosine triphosphate (ATP), so as to permit the conversion of ATP to mechanical energy by the muscles and the continuation of effort until physical exhaustion [1]. Activation of the anaerobic mechanism induces several metabolic changes such as the formation of lactic acid which triggers modifications of many variables related to the cardiorespiratory, nervous and hormonal systems, among others [1-6].
The measurement of AT has proved to be an excellent parameter for the quantification of aerobic capacity during effort, thus permitting the evaluation of the functional reserves of various systems, the cardiorespiratory one in particular, which is directly involved in the transport of $\mathrm{O}_{2}$ from atmospheric air to peripheral tissues both in healthy individuals and in patients with different diseases [1].

The AT can be obtained by invasive methods by measuring the blood concentration of lactic acid, or by noninvasive methods. Among the latter, the methods most frequently used in the laboratory involve evaluation of ventilatory variables during exercise at submaximal power levels. The gold standard for the quantification of this parameter is usually based on visual methods detecting the changes in ventilatory variables presented in paper graphs or on the computer screen itself used by one or more operators trained in this task by moving the cursor to the position corresponding to the point identifiable as the AT [1].

However, the technological advances of the last decades have brought enormous benefits by permitting the use of computerized equipment that enables the acquisition, processing and storage of a large number of respiratory and metabolic variables during physical exercise, in real time and on a respiratory cycle-to-cycle basis $[1,7]$.

Studies carried out with this new generation of equipment can be programmed using mathematical and statistical methods that permit the application of semiautomatic and/or automatic procedures for the quantification of the AT [7-10].

The objective of the present study was to compare the identification of ventilatory anaerobic threshold in healthy males using graphic visual methods and methods based on bi-segmental mathematical models applied to response of $\mathrm{CO}_{2}$ production in relation to time during dynamic physical exercise $[10,11]$.

\section{Procedures and methods}


The study was conducted on 23 healthy male volunteers aged on average $36.9 \pm 9.5$ years.

The subjects were informed about the experimental procedures and all signed an informed consent form to participate in the present study, which was approved by the Ethic Committee of University Hospital, Medical School of Ribeirão Preto, University of São Paulo, Brazil. The individuals were sedentary or practiced moderate exercise 23 times per week (walking, cycling). They were always studied 2-3 hours after a light meal.

All subjects were submitted to one or two tests of dynamic physical effort consisting of a continuous ramp type protocol, in the sitting position on an electromagnetic braking cycle ergometer coupled to a computerized ergospirometric system (CPX/D MedGraphics). In this system the tidal volume is obtained by integration of respiratory flow measured by means of a Pitot pneumotachograph, $\mathrm{O}_{2}$ concentration is measured with a zirconium electrode and $\mathrm{CO}_{2}$ concentration is measured with an infrared analyzer. The test was carried out at room temperature of about $22^{\circ} \mathrm{C}$. The use of a nose clip permitted the volunteers to breathe only through the mouthpiece.

In the protocol, the volunteer rested in position on the cycle ergometer for 1 minute and then started to pedal during a free load (3-4 Watts) period lasting 3 to 4 minutes, after which a ramp-type power was applied, individually calculated and ranging from 15 to 35 Watts/min, until physical exhaustion [1].

The ergospirometric system (CPX/D) used in the present study permits the simultaneous measurement and the calculation of many cardiorespiratory variables on a breath-to-breath basis: pulmonary ventilation (VE), $\mathrm{O}_{2}$ uptake $\left(\mathrm{VO}_{2}\right), \mathrm{CO}_{2}$ production $\left(\mathrm{VCO}_{2}\right)$, and heart rate (HR), and the power values applied and pedaling velocity on the cycle ergometer (Figure 1).

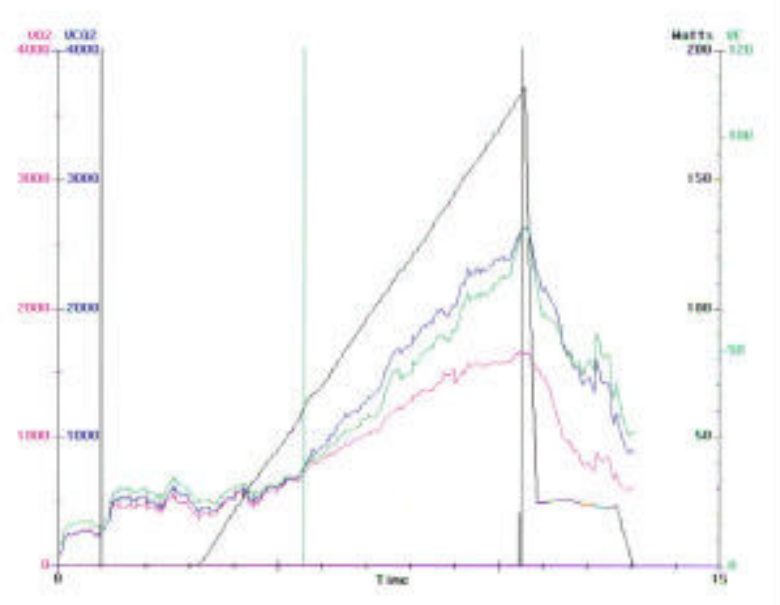

Figure 1: Graphic plot of some cardiorespiratory variables obtained during an effort test by the CPX/D system. The vertical green line corresponds to the AT point. Axis units: $\mathrm{VO}_{2}$ and $\mathrm{VCO}_{2}=\mathrm{ml} / \mathrm{min}, \mathrm{VE}=1 / \mathrm{min}$, power $=$ Watts, time $=$ minutes.
Exercise AT values, reported as $\mathrm{VO}_{2}(\mathrm{ml} / \mathrm{min})$, were calculated by two different methods which use as a criterion for the measurement of this parameter the loss of linearity of the $\mathrm{VCO}_{2}$ response in relation to time, while power and $\mathrm{VO}_{2}$ continue to increase linearly [1]. The following methods were used: 1) visual method and 2) a semi-automatic technique implemented in our Laboratory based on the use of linear-linear (Lin-Lin) and linearquadratic (Lin-Qua) bi-segmental methods for $\mathrm{VCO}_{2}$ response in relation to time [10].

The visual method expressed AT as a mean value obtained from measurements performed by three observers with proven experience in the application of the procedures used for this purpose (Figure 1). An algorithm incorporated into the CPX/D system permitted the cursor to be positioned on the point selected by the investigator on the screen of the graphic monitor.

The method of the mathematical models was based on the fitting of curves to the set of $\mathrm{VCO}_{2}$ data (80 to 160 points). The statistical methodology employed was a version of the Lerman interactive estimation procedure that comprises segmented regression models, applying the generalized least square (GLS) criterion subjected to autocorrelated errors [11]. A specific software was developed using the S-PLUS statistical package so that the algorithm divided the data into two subsets: one (S1) corresponding to the $\mathrm{VCO}_{2}$ values at the beginning of the elevation of the ramp up to an intermediate point (IP) and the other (S2) from IP to the final third of the ramp, in order to exclude the variations in $\mathrm{VCO}_{2}$ values that occur above the point of respiratory compensation [1]. The S1 and $\mathrm{S} 2$ values corresponded to the fitting of two lines in the Lin-Lin model, and to the fitting of one line (S1) and of a quadratic curve (S2) in the Lin-Qua model. In a subsequent stage, the residual sum of squares (RSS) of the model tested was calculated, with graphic printing of the RSS S1 + S2 and of the RSS of each subset (RSS S1 and RSS S2). The IP point shifted automatically from the lowest to the highest power values of the ramp, so that in the graphic representation each point expressed one RSS value for $\mathrm{S} 1+\mathrm{S} 2, \mathrm{~S} 1$ and $\mathrm{S} 2$ in the models studied (Figures 2 and 3 ).

In an initial stage, the algorithm included a test to determine whether the time series to be processed contained a minimum of twelve points, which is the limit necessary to conclude the final stage of the process. This requirement was justified by the fact that four initial points and four final points were needed to permit a quadratic adjustment (three parameters and one additional degree of freedom), in addition to four points that would permit the visualization of the graphic representation in the function criterion. These considerations explain why the initial and final points of the graphic adjustments of the criterion function present constant values - it is through this procedure that the time series of exit from routine contains the same number of points as the original 
series.

Finally, an analysis was performed on each graph to assess the possibility of identifying a minimum and single value of RRS S1 + S2, which was considered to be the point corresponding to AT, in each model studied. The mean value of $\mathrm{VO}_{2}$ at this point and at four other points (2 above and 2 below) was used to express the AT obtained from the mathematical models (the same procedure to calculate the mean was used for AT obtained by visual method).

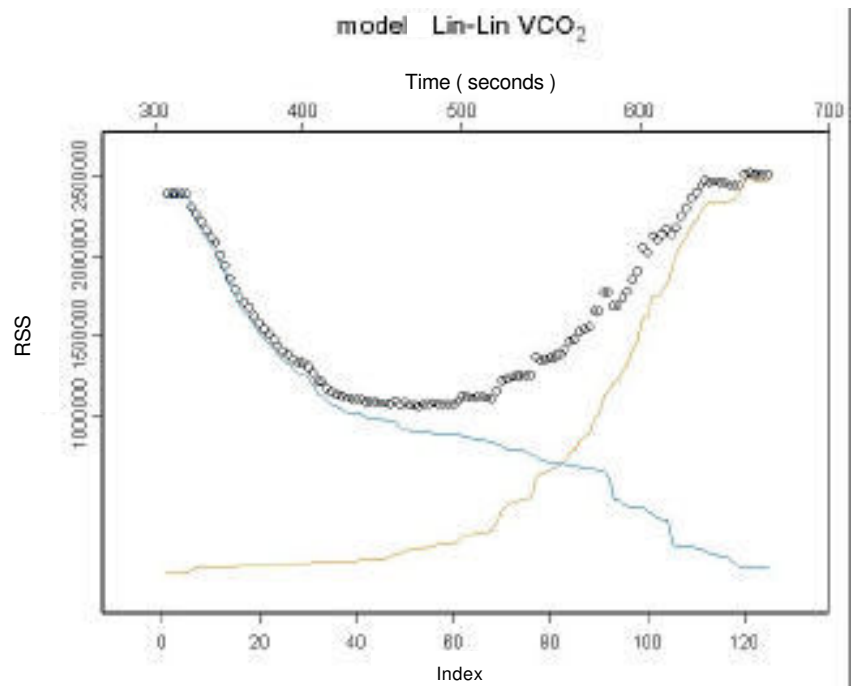

Figure 2: Lin-Lin mathematical model representing S1 + S2 (black circles), S1 (yellow line) and S2 (blue line). The Index represents the time sequence of the adjustments.

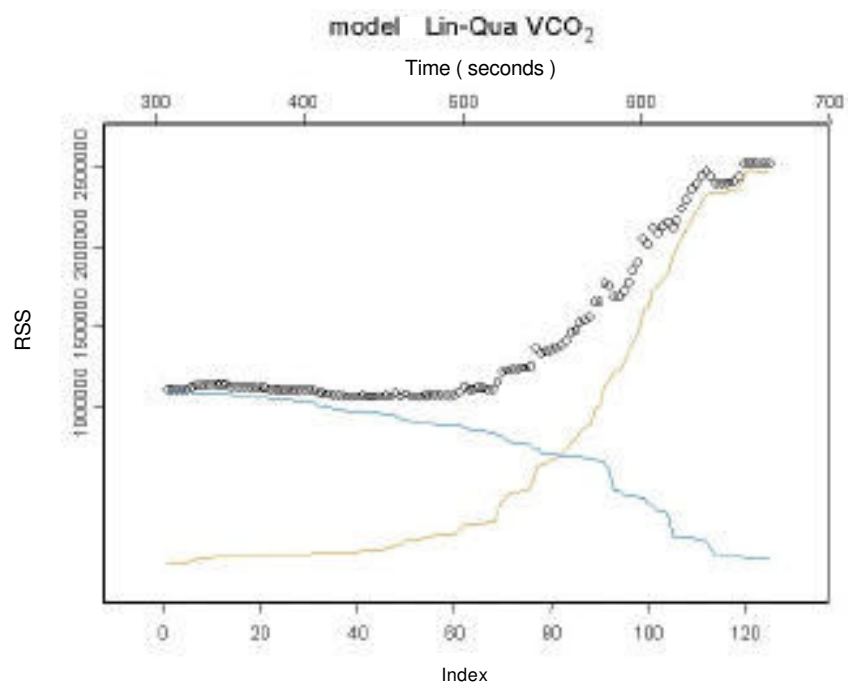

Figure 3: Lin-Qua mathematical model representing S1 + S2 (black circles), S1 (yellow line) and S2 (blue line). The Index represents the time sequence of the adjustments.

\section{Evaluation of performance of the models}

Comparative analysis of the various methods showed that the bi-segmental Lin-Qua model did not permit a reliable identification of the AT point (Figure 3) because of the wide range of minimum RSS values $(\mathrm{S} 1+\mathrm{S} 2)$. Thus, quantitative comparisons were only possible between the visual method and the linear-linear model, which always showed the presence of one or a few points in the region with lower RSS values $(\mathrm{S} 1+\mathrm{S} 2)$.

A high and significant Spearman correlation was documented for the AT values (Figure 4 ) obtained by the two methods $(r=0.82 ; p<0.0001)$. However, the values calculated by the Lin-Lin method were lower than those measured by the visual method. When the values were compared by the Wilcoxon test, the differences showed statistical significance $(\mathrm{p}<0.05)$.

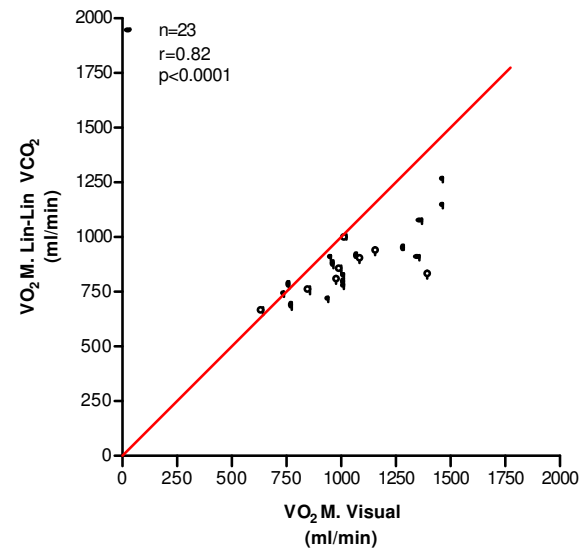

Figure 4: Correlation between the AT obtained by the visual and linear-linear methods expressed as $\mathrm{VO}_{2}(\mathrm{ml} / \mathrm{min}) . \mathrm{r}=$ Spearman correlation coefficient.

\section{Conclusions}

There was a good correlation between the AT values obtained by the visual method and by the linear-linear bisegmental model. However, the mathematical model in question underestimated the real value of AT obtained by the visual method.

So far the mathematical models tested in the present study do not show a sufficiently adequate performance to replace the visual graphic method for the quantification of the anaerobic threshold during physical exercise in men. 


\section{Acknowledgements}

The authors are grateful to FAPESP, $\mathrm{CNPq}$ and FAEPA - HCFMRP-USP for its financial support to this research project.

\section{References}

[1] Wasserman K, Hansen JE, Sue DY, Whipp BJ. Editors. Principles of Exercise Testing and Interpretation. Philadelphia: Lea \& Febiger, 1987.

[2] Nagata A, Muro M, Moritani T, Yoshida T. Anaerobic threshold determination by blood lactate and myoelectric signals. Jpn J Physiol 1981; 31:585-97.

[3] Gleim GW, Zabetakis PM, DePasquale EE, Michelis MF, Nicholas JA. Plasma osmolality, volume, and renin activity at the anaerobic threshold. J Appl Physiol 1984; 56:57-63.

[4] Rowell LB. Human Circulation. Regulation During Physical Stress. Editor. New York: Oxford University Press, 1986.

[5] Gallo Jr L, Maciel BC, Marin Neto JA, Martins LEB, Lima Filho EC, Golfetti R, Chacon MPT, Forti VAM. Control of heart rate during exercise in health and disease. Braz J Med Biol Res 1995; 28:1179-84.

[6] Marães VRFS, Silva E, Catai AM, Ribeiro TF, Gallo Jr L. The heart rate variability in dynamic exercise. Its possible role to signal anaerobic threshold. The Physiologist 2000; 43:339.
[7] Beaver WL, Wasserman K, Whipp BJ. A new method for detecting the anaerobic threshold by gas exchange. J Appl Physiol 1986; 60:2020-7.

[8] Orr GW, Green HJ, Hughson RL, Bennetett GW. A computer linear regression model to determinate ventilatory anaerobic threshold. J Appl Physiol 1982; 52: 1349-52.

[9] Green HJ, Hughson RL, Orr GW, Ranney DA. Anaerobic threshold, blood lactate and muscle metabolites in progressive exercise. J Appl Physiol 1983; 54:1032-8.

[10] Soler AM, Folledo M, Martins LEB, Lima Filho EC, Gallo $\mathrm{Jr}$ L. Anaerobic threshold estimation by statistical modeling. Braz J Med Biol Res 1989; 22: 795-7.

[11] Lerman PM. Fitting segmented regression models by gridsearch. Appl Statist 1980; 29:77-84.

Address for correspondence.

Prof. Dr. Lourenço Gallo Jr.

Cardiology Division, University Hospital of Ribeirão Preto

Av. Bandeirantes, 3900. Ribeirão Preto, SP - Brazil

Zip code: $14049-900$

lgjunior@fmrp.usp.br 